\title{
Peptide Receptor Radionuclide Therapy in Grade 3 Neuroendocrine Neoplasms: Safety and Survival Analysis in 69 Patients
}

\author{
Jingjing Zhang*, Harshad R. Kulkarni*, Aviral Singh, Karin Niepsch, Dirk Müller, and Richard P. Baum \\ THERANOSTICS Center for Molecular Radiotherapy and Precision Oncology, Zentralklinik Bad Berka, Bad Berka, Germany
}

To date, limited data are available concerning peptide receptor
radionuclide therapy (PRRT) of grade 3 (G3) neuroendocrine
neoplasms (NENs) with a Ki-67 proliferation index of greater than
$20 \%$. The purpose of this study was to analyze the long-term
outcome, efficacy, and safety of PRRT in patients with somatostatin
receptor (SSTR)-expressing G3 NENs. Methods: A total of 69
patients ( 41 men; age, $28-81$ y) received PRRT with ${ }^{177}$ Lu- or ${ }^{90}$ -
labeled somatostatin analogs (DOTATATE or DOTATOC). Twenty-
two patients had radiosensitizing chemotherapy. Kaplan-Meier analysis was performed to calculate progression-free survival (PFS) and overall survival (OS), defined from the start of PRRT, including a subgroup analysis for patients with a Ki-67 index of less than or equal to $55 \%$ and a $\mathrm{Ki}-67$ index of greater than $55 \%$. Treatment response was evaluated according to RECIST 1.1 as well as molecular imaging criteria (European Organization for Research and Treatment of Cancer). Short- and long-term toxicity was documented (Common Terminology Criteria for Adverse Events, v 5.0) using a structured database (comprising $>250$ items per patient) and retrospectively analyzed. Results: Forty-six patients had pancreatic NENs, 11 had unknown primary cancer, 6 had midgut NENs, 3 had gastric NENs, and 3 had rectal NENs. The median follow-up was 94.3 mo. The median PFS was $9.6 \mathrm{mo}$, and the median OS was 19.9 mo. For G3 NENs with a Ki-67 index of less than or equal to $55 \%(n=53)$, the median PFS was 11 mo and the median OS was 22 mo. Patients with a Ki-67 index of greater than $55 \%(n=11)$ had a median PFS of $4 \mathrm{mo}$ and a median OS of $7 \mathrm{mo}$. For patients with positive SSTR imaging but no ${ }^{18} \mathrm{~F}-\mathrm{FDG}$ uptake, the median PFS was $24 \mathrm{mo}$ and the median OS was $42 \mathrm{mo}$. A significant difference was found for both PFS and OS, with median PFS of 16 mo and 5 mo and median OS of 27 mo and 9 mo for an SUV $\max$ of greater than 15.0 and an SUV max $_{\text {ma }}$ of less than or equal to 15.0, respectively, on SSTR PET. In the group with ${ }^{18} \mathrm{~F}-\mathrm{FDG}$ uptake scored as 3 or 4 , the median PFS was $7.1 \mathrm{mo}$ and the median OS was $17.2 \mathrm{mo}$. In the group with ${ }^{18} \mathrm{~F}-\mathrm{FDG}$ uptake scored as $0-2$, the median PFS was $24.3 \mathrm{mo}$ and the median OS was $41.6 \mathrm{mo}$. PRRT was well tolerated by all patients; no grade 3 or grade 4 hematotoxicity occurred, and no clinically significant decline in renal function was observed. There was no hepatotoxicity. Conclusion: PRRT was tolerated well, without significant adverse effects, and was efficacious in G3 NENs; the clinical outcome was promising, especially in patients with a Ki-67 index of less than or equal to $55 \%$ and even in patients for whom chemotherapy had failed. Baseline ${ }^{18} \mathrm{~F}-\mathrm{FDG}$ along with SSTR

Received Jun. 22, 2018; revision accepted Aug. 7, 2018.

For correspondence or reprints contact: Richard P. Baum, THERANOSTICS Center for Molecular Radiotherapy and Precision Oncology, Zentralklinik Bad Berka, Robert-Koch-Allee 9, 99437 Bad Berka, Germany.

E-mail: richard.baum@zentralklinik.de

${ }^{*}$ Contributed equally to this work.

Published online Aug. 16, 2018.

COPYRIGHT (C 2019 by the Society of Nuclear Medicine and Molecular Imaging. molecular imaging was useful for stratifying G3 NEN patients with high uptake on SSTR PET/CT and no or minor ${ }^{18}$ F-FDG avidity-a mismatch pattern that was associated with a better long-term prognosis.

Key Words: peptide receptor radionuclide therapy; ${ }^{177} \mathrm{Lu}$; ${ }^{90}$; G3; neuroendocrine neoplasms

J Nucl Med 2019; 60:377-385

DOI: 10.2967/jnumed.118.215848

$\mathbf{N}$ euroendocrine neoplasms (NENs) are a heterogeneous group of neoplasms arising from diffuse neuroendocrine system cells (1). They are classified as grade 1 (G1), G2, and G3 according to the World Health Organization classification, which is based on mitotic activity and Ki-67 immunostaining, with a Ki-67 index of less than or equal to $2 \%, 3 \%-20 \%$, and greater than $20 \%$, respectively. High-grade (G3) NENs were previously referred to as neuroendocrine carcinomas, with an aggressive malignant character and poor prognosis; these were mainly treated with chemotherapy $(2,3)$. However, substantial heterogeneity of high-grade NENs has been observed on the basis of clinical behavior, genetic profiling, and proliferation rate. According to the World Health Organization 2017 and European Neuroendocrine Tumor Society classifications, a new G3 NEN category for the distinction of well-differentiated G3 NENs from neuroendocrine carcinomas has been introduced. Recent genetic studies also reported that G3 NENs express p53 and $\mathrm{rb1}$, which are usually negative in neuroendocrine tumors (NETs), providing a novel basis for prognostic and therapeutic stratification $(4,5)$.

During the last decade, substantial progress has been made in the treatment of G1 and G2 NENs. On the other hand, systemic cytotoxic chemotherapy, typically using platinum compounds, is usually applied for highly malignant G3 neuroendocrine carcinomas.

NENs are characterized by a high level of expression of somatostatin receptors (SSTRs), allowing the use of radiolabeled somatostatin analogs for receptor-mediated imaging and peptide receptor radionuclide therapy (PRRT) with therapeutic radioisotopes such as ${ }^{90} \mathrm{Y}$ or ${ }^{177} \mathrm{Lu}$; the latter has become an established treatment for patients with unresectable or metastatic, progressive, well-differentiated, SSTR-positive NETs (6-10). Recently, ${ }^{177} \mathrm{Lu}-$ DOTATATE (Lutathera; Advanced Accelerator Applications) was approved by both the European Commission and the U.S. Food and Drug Administration for the treatment of metastatic, progressive, well-differentiated (G1/G2), SSTR-positive gastroenteropancreatic 
TABLE 1

Demographic and Baseline Clinical Characteristics of Patients With G3 NENs $(n=69)$

\begin{tabular}{|c|c|c|}
\hline Characteristic & $\begin{array}{l}\text { No. of } \\
\text { patients }\end{array}$ & $\begin{array}{c}\% \text { of } \\
\text { patients }\end{array}$ \\
\hline \multicolumn{3}{|l|}{ Sex } \\
\hline Male & 41 & 59.4 \\
\hline Female & 28 & 40.6 \\
\hline \multicolumn{3}{|l|}{ Primary tumor site } \\
\hline Unknown & 11 & 15.9 \\
\hline Pancreas & 46 & 66.7 \\
\hline Stomach & 3 & 4.3 \\
\hline Midgut & 6 & 8.7 \\
\hline Rectum & 3 & 4.3 \\
\hline \multicolumn{3}{|l|}{ Differentiation } \\
\hline Well differentiated & 11 & 15.9 \\
\hline Moderately differentiated & 3 & 4.3 \\
\hline Poorly differentiated & 28 & 40.6 \\
\hline NA & 27 & 39.1 \\
\hline \multicolumn{3}{|l|}{ Primary tumor resected } \\
\hline Yes & 25 & 36.2 \\
\hline No & 44 & 63.8 \\
\hline \multicolumn{3}{|l|}{ Ki-67 index } \\
\hline$\leq 55 \%$ & 53 & 76.8 \\
\hline$>55 \%$ & 11 & 15.9 \\
\hline $\mathrm{NA}(>20)$ & 5 & 7.2 \\
\hline \multicolumn{3}{|l|}{ Primary treatment before PRRT } \\
\hline Somatostatin analog & 17 & 24.6 \\
\hline Chemotherapy & 35 & 50.7 \\
\hline Temozolomide & 4 & 5.8 \\
\hline Capecitabine-5-fluorouracil & 13 & 18.8 \\
\hline Streptozocin & 5 & 7.2 \\
\hline Doxorubicin & 4 & 5.8 \\
\hline Cisplatin & 14 & 20.3 \\
\hline Carboplatin & 8 & 11.6 \\
\hline Etoposide & 16 & 23.2 \\
\hline Oxaliplatin & 3 & 4.3 \\
\hline Gemcitabine & 3 & 4.3 \\
\hline $\begin{array}{l}\text { Doxorubicin- } \\
\text { cyclophosphamide- } \\
\text { vincristine }\end{array}$ & 2 & 2.9 \\
\hline $\begin{array}{l}\text { Doxorubicin-vincristine- } \\
\text { actinomycin }\end{array}$ & 1 & 1.2 \\
\hline Interferon & 2 & 2.9 \\
\hline Surgery & 34 & 49.3 \\
\hline Liver-directed therapy & 11 & 15.9 \\
\hline Radiofrequency ablation & 2 & 2.9 \\
\hline $\begin{array}{l}\text { Transarterial } \\
\text { chemoembolization }\end{array}$ & 7 & 10.1 \\
\hline Embolization & 2 & 2.9 \\
\hline $\begin{array}{l}\text { Selective internal radiation } \\
\text { therapy (radioembolization) }\end{array}$ & 5 & 7.2 \\
\hline External-beam radiotherapy & 5 & 7.2 \\
\hline $\begin{array}{l}\mathrm{NA}=\text { not available. } \\
\text { Patients were } 58.1 \pm 12.9 \text { y old. }\end{array}$ & & \\
\hline
\end{tabular}

NETs in adults. However, data on PRRT in G3 NENs are very limited.

The aim of this study was to assess the safety and efficacy, in terms of survival analysis, of PRRT in patients who have SSTRexpressing G3 NENs with a Ki-67 proliferation index of greater than $20 \%$. The prognosis of patients with distinctly different Ki-67 proliferation indices and baseline SSTR and ${ }^{18} \mathrm{~F}$-FDG PET/CT imaging was also evaluated.

\section{MATERIALS AND METHODS}

\section{Patients}

Eligible patients were adults who had histopathologically confirmed metastatic G3 NENs (Ki-67 index, >20\%) with a confirmed high level of SSTR expression-that is, tumor uptake greater than normal liver parenchyma uptake on ${ }^{68} \mathrm{Ga}$-SSTR PET imaging. Disease progression was documented within 3-6 mo before the start of PRRT.

From January 2003 to January 2017, data for a total of 69 patients who had G3 NENs (41 men and 28 women; age range, 28-81 y; mean age, 58.1 y [SD, $12.9 \mathrm{y}]$ ) and underwent PRRT were retrospectively reviewed. The study was approved by an internal review board, and written informed consent was obtained from each patient. The baseline demographics of the patients are shown in Tables 1 and 2.

Forty-six patients $(66.7 \%)$ had pancreatic NENs, and 11 (15.9\%) had unknown primary cancer, whereas the primary tumor was present in the midgut in 6 patients, in the stomach in 3 patients, and in the rectum in 3 patients. Of the 69 patients, $53(76.8 \%)$ had a Ki67 index of less than or equal to $55 \%$ and $11(15.9 \%)$ had a Ki-67 index of less than or equal to $55 \%$; for 5 patients (7.2\%), the Ki-67

TABLE 2

Baseline Imaging of Patients with G3 NENs $(n=69)$

\begin{tabular}{|c|c|c|}
\hline Characteristic & $\begin{array}{l}\text { No. of } \\
\text { patients }\end{array}$ & $\begin{array}{c}\% \text { of } \\
\text { patients }\end{array}$ \\
\hline \multicolumn{3}{|l|}{ PET imaging (baseline) } \\
\hline SSTR imaging & 69 & 100.0 \\
\hline${ }^{68} \mathrm{Ga}-\mathrm{SSTR}$ PET & 61 & 88.4 \\
\hline Octreotide scan & 8 & 11.6 \\
\hline \multicolumn{3}{|l|}{ SSTR imaging uptake } \\
\hline Grade 1 (= liver) & 0 & 0.0 \\
\hline Grade 2 (liver $<$ SUV $\left._{\max } \leq 15\right)$ & 13 & 18.8 \\
\hline Grade $3\left(15<\mathrm{SUV}_{\max }<20\right)$ & 10 & 14.5 \\
\hline Grade $4\left(S^{\prime} U V_{\max }>20\right)$ & 38 & 55.1 \\
\hline $\begin{array}{l}\text { Octreotide scan (Krenning } \\
\text { score of } \geq 3 \text { ) }\end{array}$ & 8 & 11.6 \\
\hline \multicolumn{3}{|l|}{${ }^{18} \mathrm{~F}-\mathrm{FDG}$ avidity } \\
\hline Grade 0 (none-minor uptake) & 9 & 13.0 \\
\hline Grade 1 ( $<$ liver) & 4 & 5.8 \\
\hline Grade 2 (= liver) & 1 & 1.4 \\
\hline Grade 3 (liver $<$ SUV $_{\max } \leq 10$ ) & 16 & 23.2 \\
\hline Grade $4\left(S^{\prime} U V_{\max }>10\right)$ & 29 & 42.0 \\
\hline NA & 10 & 14.5 \\
\hline $\begin{array}{l}\mathrm{NA}=\text { not available. } \\
\text { Patients were } 58.1 \pm 12.9 \text { y old. }\end{array}$ & & \\
\hline
\end{tabular}




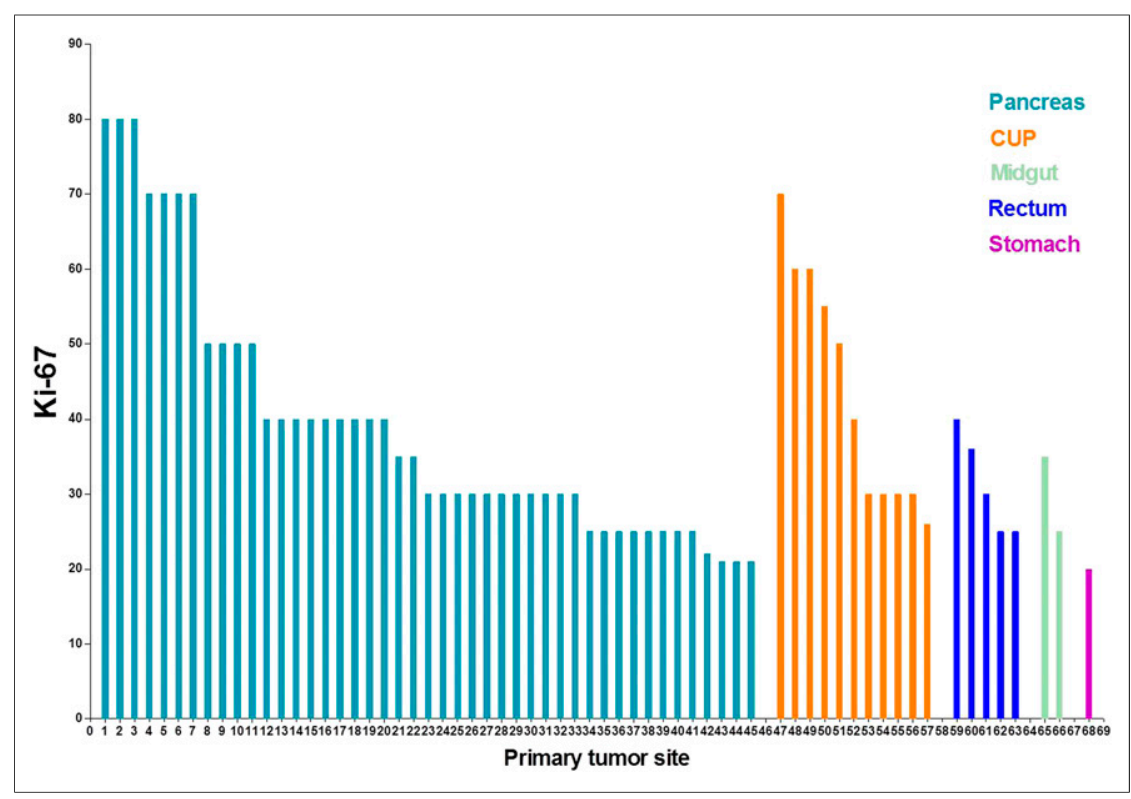

FIGURE 1. Ki-67 proliferation index for G3 NEN patients according to primary tumor site. CUP $=$ unknown primary cancer.

was unknown at the initial assessment and no samples could be obtained for reanalysis. The Ki-67 index for each patient according to the primary tumor site is shown in Figure 1.

PRRT was applied as the first-line treatment in 8 patients $(11.6 \%)$, as the second-line treatment in 25 patients $(36.2 \%)$, and as the thirdline therapy in 28 patients $(40.6 \%)$. The time span (mean \pm SD) between biopsy for the assessment of Ki-67 and the first treatment cycle was $27.1 \pm 36.8$ mo. Treatment parameters and the number of PRRT cycles are shown in Table 3 . The median administered activity for ${ }^{177} \mathrm{Lu}$ PRRT per cycle was $4.5 \pm 13.2 \mathrm{GBq}$ (range, $2.5-9.5 \mathrm{GBq}$ ). The median administered activity for ${ }^{90} \mathrm{Y}$ PRRT per cycle was $3.2 \pm$ 1.0 GBq (range, 1.3-4.8 GBq). The maximum cumulative administered activity was $38.0 \mathrm{GBq}$.

\section{Radiopharmaceutical Preparation}

The DOTA-conjugated somatostatin analogs DOTATOC, DOTANOC, and DOTATATE were labeled with ${ }^{68} \mathrm{Ga},{ }^{177} \mathrm{Lu}$, and ${ }^{90} \mathrm{Y}$, respectively, in our institutional radiopharmacy according to good manufacturing practices regulations. The radionuclide ${ }^{68} \mathrm{Ga}$ was obtained in-house from a ${ }^{68} \mathrm{Ge} /{ }^{68} \mathrm{Ga}$ generator. A highly efficient $\mathrm{NaCl}$-based ${ }^{68} \mathrm{Ga}$ labeling procedure has been developed in our hospital $(11,12) .{ }^{177} \mathrm{Lu}$ and ${ }^{90} \mathrm{Y}$ were obtained from different manufacturers. The labeling of DOTA-conjugated peptides with ${ }^{177} \mathrm{Lu}$ and ${ }^{90} \mathrm{Y}$ was performed as previously described (13). High-performance liquid chromatography was used for quality control. The radiochemical purity was always greater than $99 \%$.

\section{Treatment Regimen}

Nephroprotection was performed by amino acid infusion $(1,600 \mathrm{~mL}$ of $5 \%$ lysine $\mathrm{HCl}$ and $10 \% \mathrm{~L}$-arginine $\mathrm{HCl})(14)$. In patients with impaired renal function (glomerular filtration rate, $<60 \mathrm{~mL} / \mathrm{min}$ ) and for the application of ${ }^{90} \mathrm{Y}, 4 \%$ Gelofusine (B. Braun Melsungen AG) was infused according to patients' weights for additional nephroprotection. The infusion was started at least $30 \mathrm{~min}$ before administration of the radiotherapeutic compound and continued for $4 \mathrm{~h}$ thereafter. The radiopharmaceutical was coadministered over 10-15 min using a second infusion pump system. The activity to administer was individually chosen on the basis of the Bad Berka Score (15) — that is, the uptake in tumor lesions, as shown by ${ }^{68} \mathrm{Ga}$ SSTR PET/CT (performed before each treatment cycle), renal function, hematologic reserve, liver involvement, extrahepatic tumor burden, Ki-67 index, tumor grade, ${ }^{18} \mathrm{~F}$-FDG PET/CT status, tumor dynamics (doubling time, new lesions), weight loss, time since first diagnosis, functional activity of the tumor, previous treatments, and general status of the patient (Karnofsky Performance Scale) (16-18). The decision to use ${ }^{90} \mathrm{Y}$ or ${ }^{177} \mathrm{Lu}$ depended on the tumor mass, renal and hematologic function, previous therapy (especially chemotherapy), SUV, and other factors, as described by the Bad Berka Score $(15,19-21)$. Both radionuclides were used in subsets of patients sequentially or in combination on the same day (tandem PRRT) (15). The interval between the treatment cycles was 10-12 wk. Depending on general and hematologic status as well as tolerability, 22 patients with high uptake on ${ }^{18} \mathrm{~F}$-FDG PET/CT underwent peptide receptor chemoradionuclide therapy - that is, PRRT in combination with a low dose of capecitabine $\left(1,500 \mathrm{mg} / \mathrm{m}^{2} / \mathrm{d}\right.$ in 2 daily divided doses) for $2 \mathrm{wk}$ starting from the day of PRRT. Additionally, temozolomide was administered in 4 of 22 patients (5).

\section{Safety}

Laboratory parameters (erythrocytes, hemoglobin, platelets, leukocytes, creatinine, blood urea nitrogen, serum glutamic oxalo-acetic transaminase, serum glutamate pyruvate transaminase, bilirubin, serum alkaline phosphatase, thyroid-stimulating hormone, gamma-glutamyl transpeptidase, and respective tumor markers) were evaluated before each cycle and at restaging. Renal function was monitored by the tubular extraction rate using ${ }^{99 \mathrm{~m}} \mathrm{Tc}$-mercaptoacetyltriglycine renography; in addition, the glomerular filtration rate was determined using ${ }^{99 \mathrm{~m}} \mathrm{Tc}$-diethylenetriaminepentaacetic acid in patients with elevated serum creatinine levels. Treatment-related adverse events were graded according to the Common Terminology Criteria for Adverse Events (CTCAE, v 5.0).

Restaging was performed with SSTR PET/CT every 3-4 mo after PRRT. In case of stable disease or remission (complete or partial), restaging was performed with SSTR PET/CT every 6 mo until disease progression was evident on imaging. SSTR PET/CT and ${ }^{18} \mathrm{~F}-\mathrm{FDG}$ PET/CT were performed until January 2014 with a Siemens Biograph Duo and since then with a Siemens Biograph mCT Flow 64. SUV $\max$ were obtained by drawing circular regions of interest, which were automatically adapted ( $40 \%$ isocontour) to a 3-dimensional volume of interest using commercial software provided by the vendor. Contrastenhanced CT (spiral CT using a Biograph mCT Flow 64) was acquired after the intravenous administration of $60-100 \mathrm{~mL}$ of nonionic iodinated contrast agent. Images were evaluated by 2 experienced nuclear medicine specialists. MRI was performed in selected cases (allergy to iodinated contrast agent or poor detectability of liver metastases on CT scan), and an abdominal ultrasound was obtained at each visit. PRRT was resumed if progression occurred after a therapy interval of more than 6 mo (so-called next "treatment phase" of PRRT).

\section{Response Assessment}

The treatment response was assessed on CT or MR images according to RECIST 1.1 (22). Imaging was performed before each PRRT cycle and at restaging. The disease control rate was defined 
TABLE 3

Treatment Cycles and Cumulative Administered Radioactivity for PRRT with ${ }^{177} \mathrm{Lu}$, ${ }^{90}$ Y, and Both Radionuclides $(n=69)$

\begin{tabular}{|c|c|c|c|c|}
\hline \multirow[b]{2}{*}{ No. of treatment cycles } & \multirow[b]{2}{*}{ No. of patients } & \multirow[b]{2}{*}{$\%$ of patients } & \multicolumn{2}{|c|}{$\begin{array}{l}\text { Cumulative radioactivity } \\
\text { for } 69 \text { patients }\end{array}$} \\
\hline & & & Mean & SD \\
\hline \multicolumn{5}{|l|}{ PRRT (69 patients) } \\
\hline 1 & 13 & 18.8 & 4.3 & 2.0 \\
\hline 2 & 16 & 23.2 & 11.6 & 3.9 \\
\hline 3 & 12 & 17.4 & 16.0 & 5.4 \\
\hline 4 & 9 & 13.0 & 21.0 & 6.3 \\
\hline 5 & 10 & 14.5 & 30.3 & 5.6 \\
\hline 6 & 6 & 8.7 & 32.1 & 9.5 \\
\hline 7 & 2 & 2.9 & 38.2 & 2.4 \\
\hline 8 & 1 & 1.4 & 46.9 & / \\
\hline \multicolumn{5}{|l|}{ PRRT with ${ }^{177} \mathrm{Lu}$ (29 patients) } \\
\hline 1 & 6 & 8.7 & 6.2 & 2.1 \\
\hline 2 & 9 & 13.0 & 13.6 & 4.7 \\
\hline 3 & 5 & 7.2 & 21.0 & 7.8 \\
\hline 4 & 3 & 4.3 & 23.7 & 9.9 \\
\hline 5 & 5 & 7.2 & 33.5 & 11.8 \\
\hline 6 & 1 & 1.4 & 41.7 & \\
\hline 7 & 0 & 0.0 & 0.0 & 0.0 \\
\hline 8 & 0 & 0.0 & 0.0 & 0.0 \\
\hline \multicolumn{5}{|l|}{ PRRT with ${ }^{90} Y$ (16 patients) } \\
\hline 1 & 7 & 10.1 & 3.2 & 1.2 \\
\hline 2 & 4 & 5.8 & 7.4 & 2.7 \\
\hline 3 & 3 & 4.3 & 9.5 & 3.7 \\
\hline 4 & 1 & 1.4 & 7.3 & \\
\hline 5 & 0 & 0.0 & 0.0 & 0.0 \\
\hline 6 & 1 & 1.4 & 19.0 & \\
\hline 7 & 0 & 0.0 & 0.0 & 0.0 \\
\hline 8 & 0 & 0.0 & 0.0 & 0.0 \\
\hline \multicolumn{5}{|c|}{ PRRT with ${ }^{177} \mathrm{Lu}$ and ${ }^{90} \mathrm{Y}$ (24 patients) } \\
\hline 2 & 3 & 4.3 & 10.2 & 4.8 \\
\hline 3 & 4 & 5.8 & 14.6 & 5.8 \\
\hline 4 & 5 & 7.2 & 22.2 & 8.6 \\
\hline 5 & 5 & 7.2 & 27.0 & 10.6 \\
\hline 6 & 4 & 5.8 & 33.0 & 13.9 \\
\hline 7 & 2 & 2.9 & 38.2 & \\
\hline 8 & 1 & 1.4 & 46.9 & \\
\hline
\end{tabular}

as complete remission, partial remission (PR), and stable disease. The best objective response rate was defined as patients achieving complete remission or PR at follow-up according to RECIST. The molecular response was evaluated according to European Organization for Research and Treatment of Cancer criteria (23).

\section{Statistical Analysis}

A Kaplan-Meier survival analysis was performed to calculate progression-free survival (PFS) and overall survival (OS), defined from the start of PRRT. The log-rank test and the Cox proportional hazards model were used to analyze the survival distribution of subgroups.
Continuous variables were denoted as mean \pm SD. Differences between 2 independent groups were determined by Student $t$ tests. Differences among groups were compared with 1-way ANOVAs. All statistical tests were 2-tailed, and a $P$ value of less than 0.05 was considered statistically significant.

\section{RESULTS}

\section{Safety}

No CTCAE grade 4 hematotoxicity was observed, and except for 1 patient $(1.4 \%)$ with grade 3 leukocytopenia, there was no 
significant toxicity. No grade 3 or 4 anemia was observed after PRRT, although 2 patients $(2.9 \%)$ had grade 3 anemia before PRRT. No myelodysplastic syndrome or leukemia occurred during follow-up until death or the study cutoff date.

No CTCAE grade 3 or 4 nephrotoxicity was observed in any patient. At baseline, creatinine was normal in 57 of 69 patients $(82.6 \%)$, whereas $14.5 \%(10 / 69)$ had grade 1 and $2.9 \%(2 / 69)$ had grade 2 renal dysfunction. On follow-up, $84.6 \%$ (44/52) had normal creatinine, $13.5 \%$ (7/52) had grade 1 elevation of creatinine, and $1.9 \%$ (1/52) had grade 2 elevation of creatinine, respectively. However, there was no correlation with the number of cycles or the cumulative administered radioactivity. No patients with grade 2 renal impairment at baseline demonstrated a further decline in renal function. The glomerular filtration rate did not show any significant change after PRRT, despite the fact that some patients had grade 2 renal impairment at baseline (glomerular filtration rate, $35-60 \mathrm{~mL} / \mathrm{min} / 1.73 \mathrm{~m}^{2}$ ). No liver function abnormality was observed after therapy.

\section{Treatment Response}

Response evaluation was possible in 55 patients. Five patients died shortly after the first PRRT cycle, and in 9 patients the clinical condition deteriorated severely, preventing repeated imaging. According to RECIST 1.1, the disease control rate at 3 mo after PRRT was $78.2 \%$, including $30.9 \%$ of patients with PR and $47.3 \%$ with stable disease. The molecular imaging responses at 3 mo after PRRT were $49.1 \%$ with PR, $21.8 \%$ with stable disease, $27.3 \%$ with progressive disease, and $5.5 \%$ with a mixed response. The treatment responses are shown in Table 4.

\section{Survival}

Until the study cutoff date in January 2018, 56 of 69 patients ( $81 \%$ ) with G3 NENs died. The median follow-up time was 94.3 mo (interquartile range, 56.2-104.9). For the entire group of 69 patients, the median PFS was 9.6 mo and the median OS was 19.9 mo. Among the 64 patients with G3 NENs (excluding 5 patients in whom the exact Ki-67 index was not known), the median PFS for a Ki-67 index of less than or equal to 55\% was $11 \mathrm{mo}(n=53)$ and the median PFS for a Ki-67 index of greater than $55 \%$ was 4 mo $(n=11)(P<0.05)$ (Fig. 2$)$. The median OS for a Ki-67 index of less than or equal to $55 \%$ was 22 mo, and the median OS for a Ki-67 index of greater than $55 \%$ was 7 mo $(P<0.05)$ (Fig. 3).

Peptide receptor chemoradionuclide therapy was performed in 22 patients; 18 patients received capecitabine, and 4 patients received temozolomide. In this group, the median PFS was 9.8 mo and the median OS was 21.6 mo.

\section{PET Imaging Related to Response and Survival}

Of the 55 patients for whom a response assessment was performed, $86.0 \%$ (37/43) demonstrating an objective response had an $\mathrm{SUV}_{\max }$ of greater than 15.0 on baseline ${ }^{68} \mathrm{Ga}-\mathrm{SSTR}$ PET/ CT. All 6 patients with an $\mathrm{SUV}_{\max }$ of greater than 50 had an objective response to PRRT (4 achieving PR and 2 with stable disease) (Fig. 4A). The PFS and OS based on the study-specified ${ }^{68}$ Ga-SSTR PET/CT SUV $\max$ cutoff of 15.0 are shown in Figure 4B. A significant difference was found for both PFS and OS, with median PFS of 16 mo and $5 \mathrm{mo}(P<0.05)$ and median OS of 27 mo and 9 mo $(P<0.05)$ for an $\mathrm{SUV}_{\max }$ of greater than 15.0 and an $\mathrm{SUV}_{\max }$ of less than or equal to 15.0 , respectively.

In 48 of 59 patients having baseline ${ }^{18} \mathrm{~F}$-FDG PET/CT, the responses were also evaluated. All 9 patients with grade 0-2 imaging results (no or very mild ${ }^{18} \mathrm{~F}-\mathrm{FDG}$ uptake, less than that in the liver, mismatch between SSTR and ${ }^{18}$ F-FDG imaging) had an objective response to PRRT at 3 mo ( 7 achieving PR and 2 with stable disease). On the other hand, of the 39 patients with grade 3 or 4 status (uptake higher than that in the liver), 6 had PR, 23 had stable disease, and 10 had progressive

TABLE 4

Treatment Response at 3 Months After PRRT $(n=55)$

\begin{tabular}{|c|c|c|c|c|c|c|}
\hline \multirow{2}{*}{$\begin{array}{c}\text { Response at } 3 \\
\text { mo after PRRT } \\
\text { in } 55 \text { patients }\end{array}$} & \multicolumn{2}{|c|}{$\begin{array}{c}\text { Ki-67 index of } \leq 55 \% \\
(n=50)\end{array}$} & \multicolumn{2}{|c|}{$\begin{array}{c}\text { Ki-67 index of }>55 \% \\
(n=5)\end{array}$} & \multicolumn{2}{|c|}{ Total $(n=55)$} \\
\hline & No. of patients & $\%$ of patients & No. of patients & $\%$ of patients & No. of patients & $\%$ of patients \\
\hline \multicolumn{7}{|c|}{ SSTR imaging response } \\
\hline $\mathrm{CR}$ & 0 & 0 & 0 & 0 & 0 & 0 \\
\hline PR & 26 & 52 & 1 & 20 & 27 & 49.1 \\
\hline Stable disease & 11 & 22 & 1 & 20 & 12 & 21.8 \\
\hline PD & 12 & 24 & 3 & 60 & 15 & 27.3 \\
\hline Mixed response & 3 & 6 & 0 & 0 & 3 & 5.5 \\
\hline DCR & 37 & 74 & 2 & 40 & 39 & 70.9 \\
\hline \multicolumn{7}{|l|}{ CT or MRI response } \\
\hline CR & 0 & 0 & 0 & 0 & 0 & 0 \\
\hline PR & 17 & 34 & 0 & 0 & 17 & 30.9 \\
\hline Stable disease & 24 & 48 & 2 & 40 & 26 & 47.3 \\
\hline PD & 9 & 18 & 3 & 60 & 12 & 21.8 \\
\hline DCR & 41 & 82 & 2 & 40 & 43 & 78.2 \\
\hline
\end{tabular}

$\mathrm{CR}=$ complete remission; $\mathrm{PD}=$ progressive disease; $\mathrm{DCR}=$ disease control rate. 


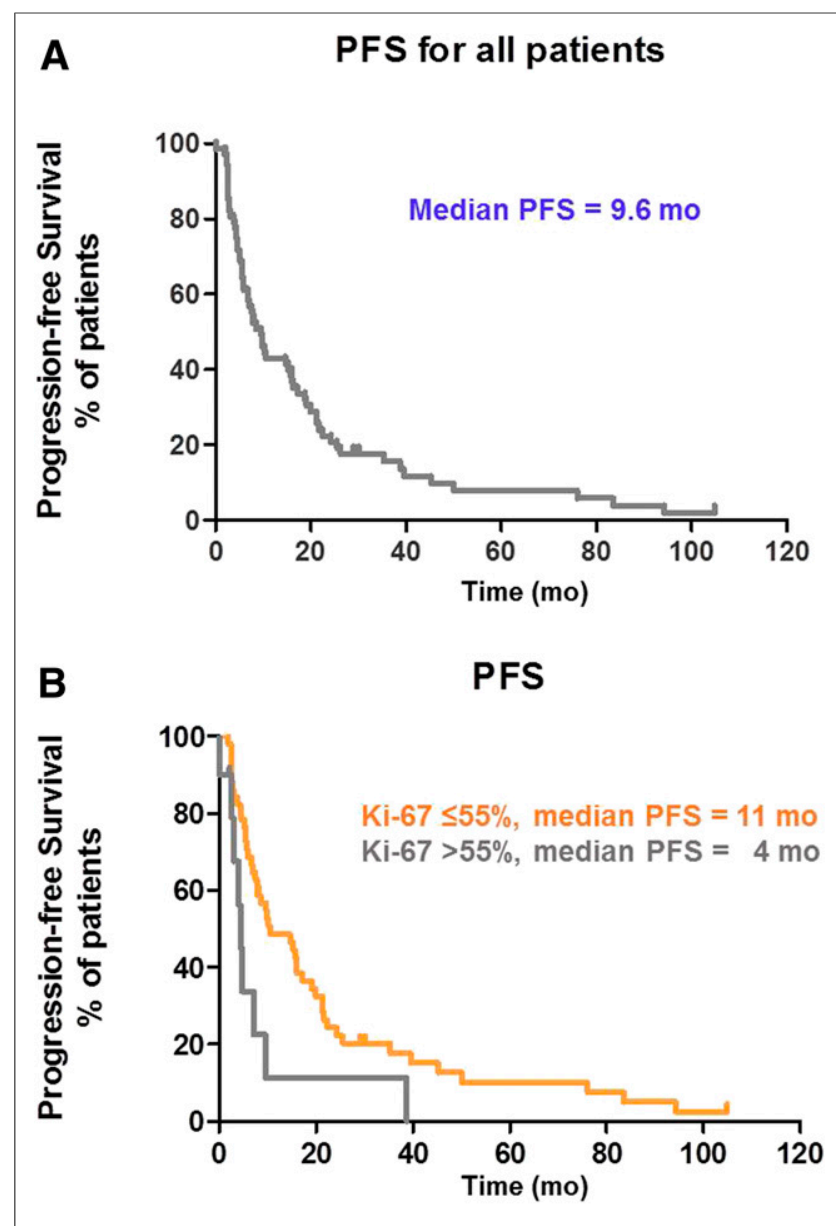

FIGURE 2. Kaplan-Meier curves for PFS (in months) from start of PRRT for all patients with $\mathrm{Ki}-67$ index of $>20 \%$ (A) and for subgroups (B) with Ki-67 index of $\leq 55 \%$, and $\mathrm{Ki}-67$ index of $>55 \%$.

disease. In the group with ${ }^{18} \mathrm{~F}$-FDG uptake scored as 3 or $4(n=$ 45), the median PFS was 7.1 mo and the median OS was 17.2 mo. In the group with ${ }^{18}$ F-FDG uptake scored as $0-2(n=$ 14), the median PFS was 24.3 mo and the median OS was 41.6 mo (Fig. 5).

\section{DISCUSSION}

Most PRRT studies have focused on G1 and G2 NENs (6-8,2427). The NETTER-1 study, a prospective randomized controlled phase 3 clinical trial using tandem treatment with ${ }^{177} \mathrm{Lu}$-DOTATATE and octreotide (long-acting release) $(30 \mathrm{mg})$ in patients with advanced midgut neuroendocrine tumors, also focused on well-differentiated NENs; in that study, a markedly improved PFS and a significantly higher response rate were reported with the tandem treatment than with high-dose octreotide (long-acting release) (6). Clinical experience with PRRT in patients with G3 NENs is limited, even though SSTR expression, in principle, enables the use of a ${ }^{177} \mathrm{Lu}$ - or ${ }^{90} \mathrm{Y}$-coupled somatostatin analog as a therapy option (Figs. 6 and 7). Only a few PRRT studies have included patients with G3 NENs or NENs with a high proliferation index $(5,28-30)$.

To date, this was the largest reported cohort of patients with SSTR-expressing G3 NENs treated with PRRT. Although this patient group was heterogeneous, including PRRT combined with radiosensitizing chemotherapy in some patients, most G3 NEN patients received only PRRT after the failure of prior chemotherapy. The follow-up (median, $94.3 \mathrm{mo}$ ) in this patient cohort was the longest among all published studies.

The median PFS and OS of patients with G3 NENs were significantly shorter than those of patients with well-differentiated G1 and G2 NENs $(6,31)$. However, compared with the results and the PFS and OS obtained with other treatment modalities in G3 NEN patients, our results were very encouraging. In the NORDIC study, a median PFS of just 4 mo and a median OS of only 11 mo were reported for patients who had a World Health Organization classification of G3 and received first-line chemotherapy (32). With a median PFS of 9.6 mo and a median OS of 19.9 mo, our patient cohort treated with PRRT had twice-longer PFS and relatively longer OS; these findings were even more promising because $76.7 \%$ of our patients had already received at least 1 line of treatment before PRRT. Our results match well with those of a single-center study in 28 patients from Australia (5), for whom a PFS of 9 mo and a median OS of 19 mo were reported.

The median OS obtained for patients with a Ki-67 index of less than or equal to $55 \%$ in the present study was markedly longer than that reported by the NORDIC group ( 22 vs. 14 mo). These results suggest that, compared with platinum-based chemotherapy,

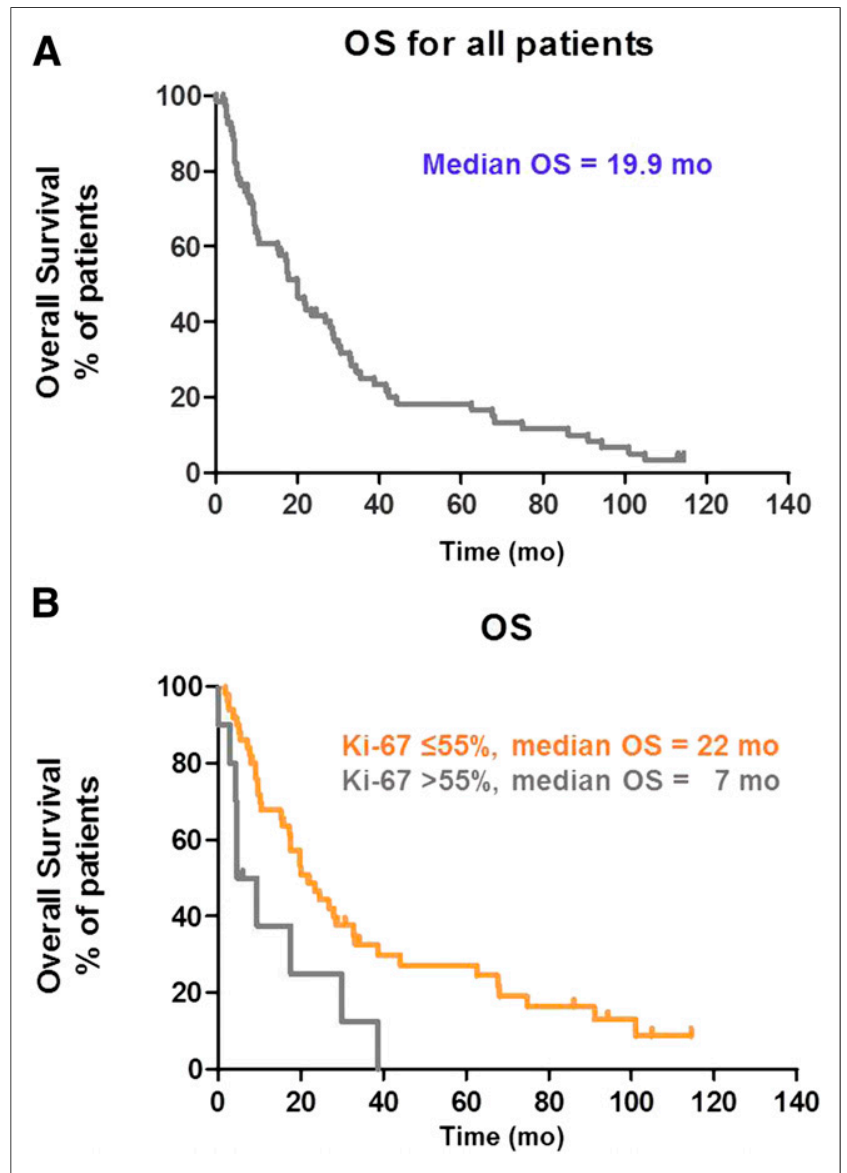

FIGURE 3. Kaplan-Meier curves for OS (in months) from start of PRRT for all patients with $\mathrm{Ki}-67$ index of $>20 \%$ (A) and for subgroups (B) with $\mathrm{Ki}-67$ index of $\leq 55 \%$, and $\mathrm{Ki}-67$ index of $>55 \%$. 


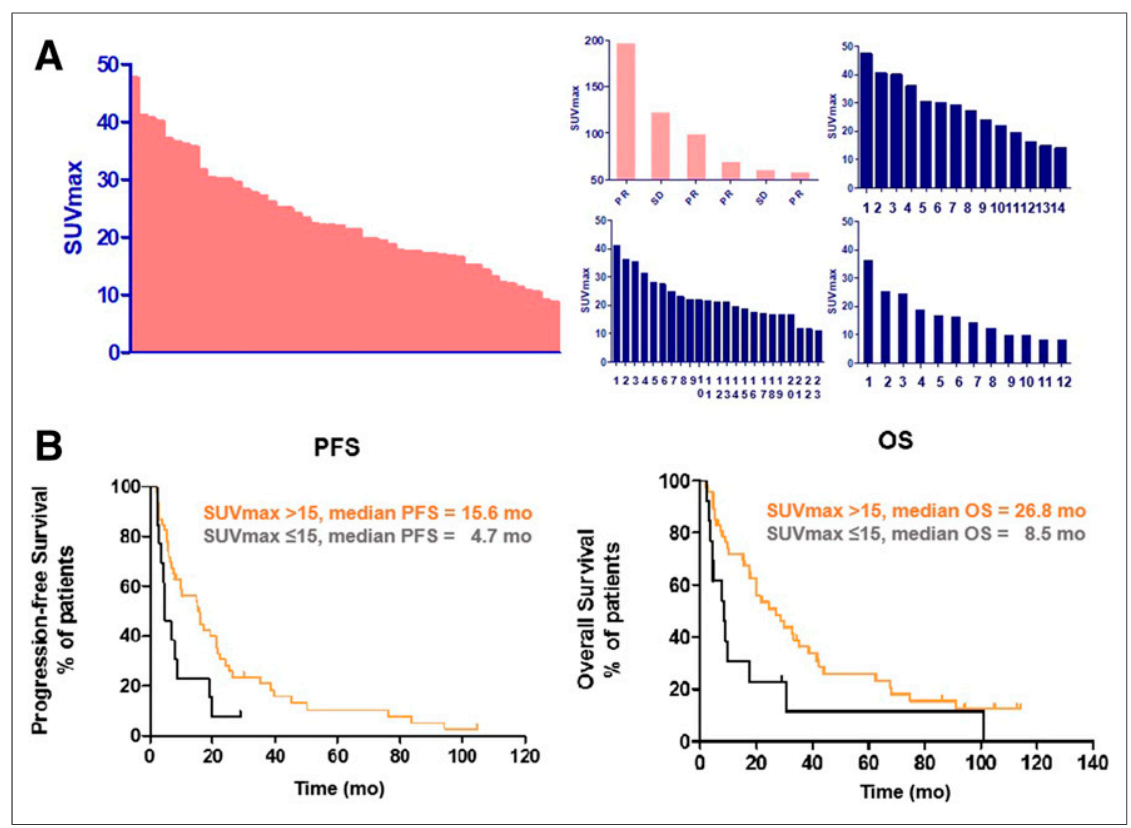

FIGURE 4. (A) Distribution of SUV ${ }_{\max }$ on ${ }^{68} \mathrm{Ga}-\mathrm{SSTR}$ PET before PRRT (red). Distribution of $\mathrm{SUV}_{\max }$ at baseline in subgroups (blue) that had PR (top right), stable disease (SD) (bottom left), and progressive disease (bottom right) at 3 mo after PRRT. (B) Kaplan-Meier curves for PFS and OS in subgroups with

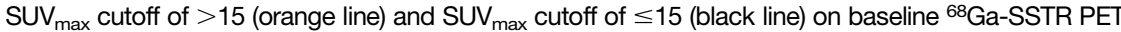
imaging.

PRRT may be a superior first-line therapeutic option in selected patients with a high level of SSTR expression and a Ki-67 index of less than or equal to $55 \%$. On the contrary, in patients with a $\mathrm{Ki}-67$ index of greater than $55 \%$, the median OS was longer for chemotherapy (7 vs. $10 \mathrm{mo}$ ). Therefore, platinum-based chemotherapy may be a better first-line therapy for patients with a $\mathrm{Ki}$ 67 index of greater than 55\%. The median OS obtained in our patients with a Ki-67 index of less than or equal to $55 \%$ was markedly shorter than that in the Australian study (22 vs. 46 mo);
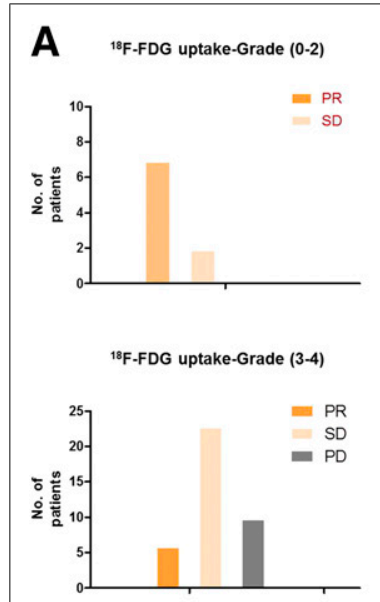
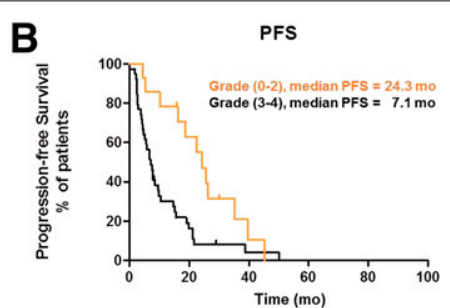

os

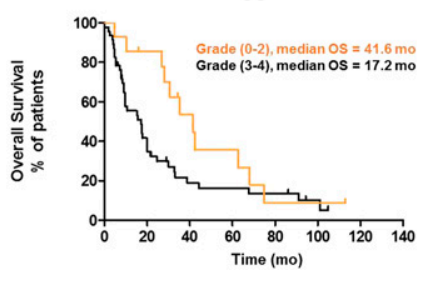

FIGURE 5. (A) Response at 3 mo after PRRT in subgroups (patients with grade $0-2$ and patients with grade 3 or 4 ) with ${ }^{18} \mathrm{~F}-\mathrm{FDG}$ PET at baseline. PD = progressive disease. (B) Kaplan-Meier curves for PFS and $\mathrm{OS}$ in subgroups with grade $0-2$ (orange line) and grade 3 or 4 (black line) on baseline ${ }^{18} \mathrm{~F}-\mathrm{FDG}$ PET. the fact that the number of patients in the present study was significantly larger (53 vs. 22) contributed to an even greater heterogeneity among the patient population. Indeed, our results for patients with a Ki-67 index of greater than $55 \%$ matched those of the Australian study (7 mo in both studies).

We also found a significant difference between the PFS values for the 2 groups$11 \mathrm{mo}$ for a $\mathrm{Ki}-67$ index of less than or equal to $55 \%(n=53)$ and 4 mo for a Ki-67 index of greater than 55\% $(n=$ 11) - a trend similar to that in a recent Italian study (29) in which patients who had a Ki-67 index of greater than $35 \%$ were compared with patients who had a Ki-67 index of less than $35 \%$; in that study, the latter patients had a significantly longer PFS (6.8 and $26.3 \mathrm{mo}$, respectively) $(P=0.005)$. The difference in the absolute PFS in the present study could be attributed to the lower cutoff for Ki-67 (of 35\%) in the Italian study. In addition, the authors of the

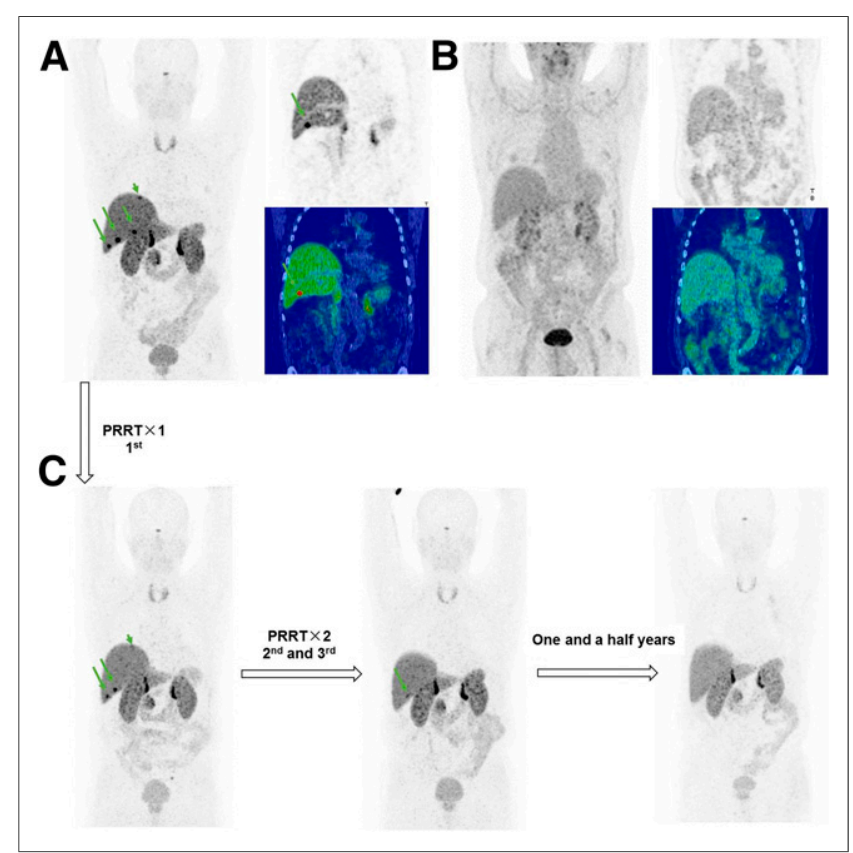

FIGURE 6. 71-y-old woman with metastatic pancreatic NENs and Ki-67 index of $25 \%$. Patient had undergone pancreatic tail resection with splenectomy and adhesiolysis and showed disease progression after 8 cycles of chemotherapy with cisplatin and etoposide within 6-mo period. (A) Maximum-intensity-projection (MIP) (left) and coronal (right) images from ${ }^{68} \mathrm{Ga}$-SSTR PET/CT showed SSTR expression (arrows) in liver metastases with $\mathrm{SUV}_{\max }$ of 40.0. (B) MIP (left) and coronal (right) images from ${ }^{18} \mathrm{~F}$-FDG PET/CT showed no significant ${ }^{18} \mathrm{~F}-\mathrm{FDG}$ hypermetabolism in liver metastases and complete mismatch between ${ }^{8} \mathrm{Ga}-\mathrm{SSTR}$ and ${ }^{18} \mathrm{~F}-\mathrm{FDG}$ at baseline. Patient was treated with 3 cycles of ${ }^{177} \mathrm{Lu}-\mathrm{DOTA}-$ TOC PRRT with cumulative administered radioactivity of $21.8 \mathrm{GBq}$. (C) Restaging scans showed significant response in liver metastases at $3 \mathrm{mo}$ (PR) (left) and complete remission on longer follow-up (middle and right), with progression-free interval of $32 \mathrm{mo}$ from first cycle of PRRT. 


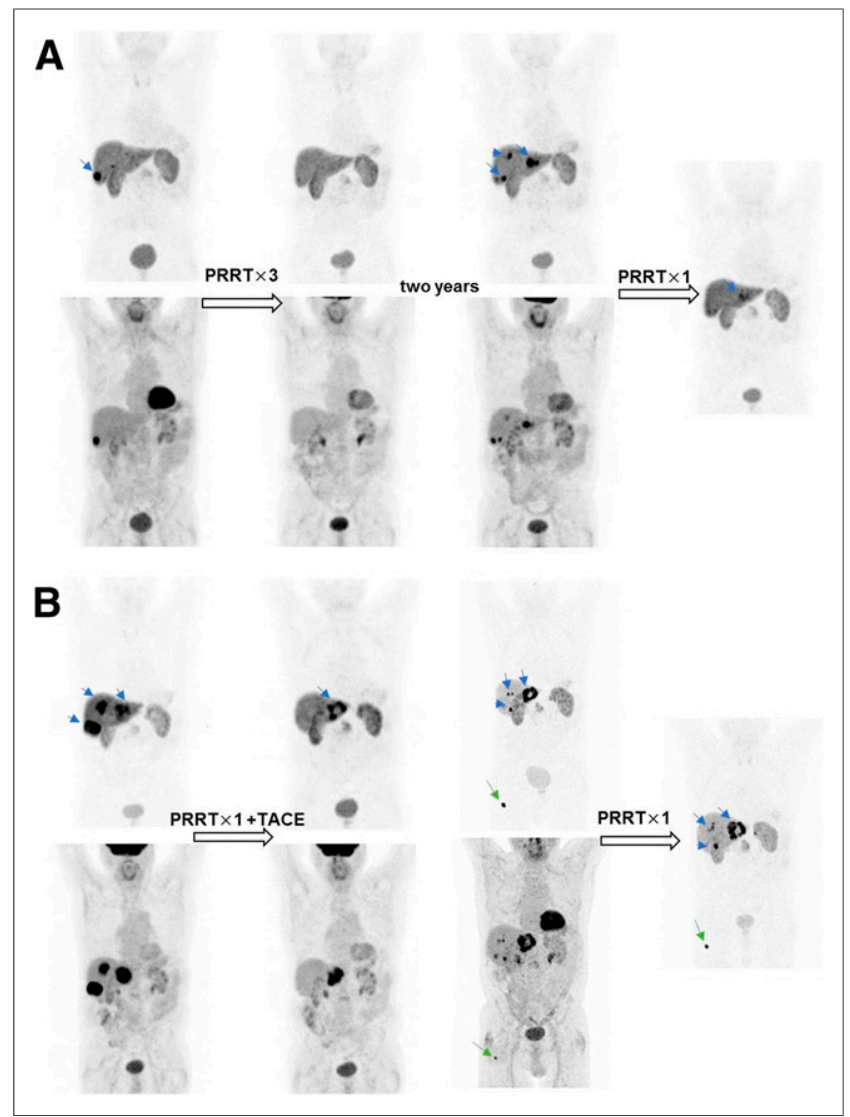

FIGURE 7. Serial ${ }^{88} \mathrm{Ga}$-SSTR PET/CT and ${ }^{18} \mathrm{~F}-\mathrm{FDG}$ PET/CT maximumintensity-projection (MIP) images of 77-y-old man with pancreatic NENs and liver metastases and with $\mathrm{Ki}-67$ proliferation index of $30 \%$ for primary tumor. Previous treatments (from 2006 to 2008) were left pancreatectomy, splenectomy, atypical partial gastrectomy, resection of left colonic flexure, liver metastasis resection and cholecystectomy, gastric fundus resection, liver metastasis radiofrequency ablation, and peritoneal adhesiolysis. (A) Baseline ${ }^{68} \mathrm{Ga}-\mathrm{SSTR}$ PET/CT (left) showed SSTR expression in liver metastases with SUV $V_{\max }$ of 26.0 , and significant hypermetabolism was noted on ${ }^{18} \mathrm{~F}-\mathrm{FDG}$ PET/CT. After 3 cycles of PRRT, good response $(\mathrm{PR})$ with significant reduction of hepatic tumor burden was seen on both ${ }^{68} \mathrm{Ga}-\mathrm{SSTR}$ and ${ }^{18} \mathrm{~F}-\mathrm{FDG}$ PET/CT. This disease was stable, with progression-free interval of $32 \mathrm{mo}$. Patient progressed in 2011 with multiple liver metastases (blue arrows) and was further treated with 1 cycle of ${ }^{177}$ Lu-DOTATATE PRRT; once again, significant regression of hepatic metastases was seen (right). (B) After PFS of 19 mo, patient progressed in 2013 with multiple liver metastases (left), apparent as match between ${ }^{68} \mathrm{Ga}$-SSTR and ${ }^{18} \mathrm{~F}-\mathrm{FDG}$ PET/CT. He then underwent combined treatment with ${ }^{177}$ Lu-DOTATATE PRRT and transarterial chemoembolization (TACE). Restaging scan showed marked PR in hepatic metastases. After short PFS of $10 \mathrm{mo}$, new bone metastases in right femur were detected on both ${ }^{18} \mathrm{~F}-\mathrm{FDG}$ and ${ }^{68} \mathrm{Ga}$-DOTATATE PET/ CT (green arrows), and patient was treated with another cycle of PRRT in 2015. In all, this patient received 6 cycles of PRRT with cumulative administered radioactivity of $24.5 \mathrm{GBq}$ of ${ }^{177}$ Lu-DOTATATE/TOC and $7.5 \mathrm{GBq}$ of ${ }^{90} \mathrm{Y}$-DOTATATE before he died of disease progression $7.5 \mathrm{y}$ from first cycle of PRRT.

Italian study included patients with a Ki-67 index of greater than $15 \%$ - that is, the "gray zone" (Ki-67 index of 15\%-20\%), defined by the European Neuroendocrine Tumor Society as G2 tumors (29).

Indeed, Ki-67 alone may not be truly representative of the tumor grade, mainly because the heterogeneity of different metastases yields different values from biopsies of different lesions. ${ }^{18}$ F-FDG PET/CT plays an important role in the prognosis of NETs, with an ${ }^{18} \mathrm{~F}$-FDG-positive tumor denoting a more aggressive phenotype (33). We characterized the ${ }^{18}$ F-FDG imaging phenotypes according to the grades of uptake. Patients with no or very faint ${ }^{18} \mathrm{~F}$-FDG uptake responded well to PRRT at 3 mo-that is, a pattern of mismatch between SSTR and ${ }^{18} \mathrm{~F}-\mathrm{FDG}$ imaging is of prognostic relevance. The median PFS for the group with no to mild ${ }^{18} \mathrm{~F}-\mathrm{FDG}$ uptake was significantly longer than that for the highly ${ }^{18} \mathrm{~F}-\mathrm{FDG}-$ avid group. These results are concordant with those of a previous study examining the role of ${ }^{18} \mathrm{~F}-\mathrm{FDG}$ PET/CT in advanced, well-differentiated grade 1 or 2 NETs (34).

${ }^{68} \mathrm{Ga}$-SSTR PET/CT may also play an important role in predicting the response to PRRT in G3 NENs. Patients with a good response and a favorable outcome after PRRT had a relatively higher $\mathrm{SUV}_{\max }$ on SSTR PET/CT imaging. This finding is in line with those of another study, in which all grades of NENs were included (35). When a cutoff of 15.0 was used for the ${ }^{68} \mathrm{Ga}$-SSTR PET $\mathrm{SUV}_{\text {max }}$, a significant association with both PFS and OS was observed.

A limitation of the present study is that it was a retrospective analysis. There were variations in SSTR affinities because different radiopharmaceuticals were used. Also, the number of patients with a Ki-67 index of greater than 55\% was limited, probably because of the lack of SSTR expression in this group. The fact that 13 patients received only 1 PRRT cycle may have influenced the prognosis. Another limitation is the lack of availability of the exact Ki-67 index in 5 patients; however, these patients were referred from other centers and were characterized as having histopathologically confirmed G3 NENs. Furthermore, almost one-third $(31.9 \%)$ of the patients with a high-grade ${ }^{18} \mathrm{~F}$-FDG PET/ CT phenotype and a more aggressive tumor type received peptide receptor chemoradionuclide therapy, and the potential additional value of the concomitant radiosensitizing chemotherapy is unclear. Further randomized and controlled studies certainly remain warranted.

\section{CONCLUSION}

PRRT was efficacious in patients with G3 neuroendocrine neoplasms, even those for whom chemotherapy had failed, resulting in a promising clinical outcome-especially for patients with a Ki-67 index of less than or equal to 55\%. A high SUV on SSTR PET/CT and no or minor ${ }^{18} \mathrm{~F}-\mathrm{FDG}$ avidity were associated with a better prognosis-that is, ${ }^{18} \mathrm{~F}-\mathrm{FDG}$ PET/ CT along with SSTR PET/CT helped to stratify patients with G3 NENs. PRRT was well tolerated, without significant adverse effects.

\section{DISCLOSURE}

No potential conflict of interest relevant to this article was reported.

\section{ACKNOWLEDGMENTS}

We thank the radiopharmacists, radiochemists, physician colleagues, nursing staff, and nuclear medicine technologists at Zentralklinik Bad Berka for their support. 


\section{REFERENCES}

1. Modlin IM, Oberg K, Chung DC, et al. Gastroenteropancreatic neuroendocrine tumours. Lancet Oncol. 2008;9:61-72.

2. Fazio N, Spada F, Giovannini M. Chemotherapy in gastroenteropancreatic (GEP) neuroendocrine carcinomas (NEC): a critical view. Cancer Treat Rev. 2013;39:270-274.

3. Angelousi A, Kaltsas G, Koumarianou A, Weickert MO, Grossman A. Chemotherapy in NETs: when and how. Rev Endocr Metab Disord. 2017;18:485-497.

4. Tang LH, Basturk O, Sue JJ, Klimstra DS. A practical approach to the classification of WHO grade 3 (G3) well differentiated neuroendocrine tumor (WD-NET) and poorly differentiated neuroendocrine carcinoma (PD-NEC) of the pancreas. Am J Surg Pathol. 2016;40:1192-1202.

5. Thang SP, Lung MS, Kong G, et al. Peptide receptor radionuclide therapy (PRRT) in European Neuroendocrine Tumour Society (ENETS) grade 3 (G3) neuroendocrine neoplasia (NEN): a single-institution retrospective analysis. Eur J Nucl Med Mol Imaging. 2018;45:262-277.

6. Strosberg J, El-Haddad G, Wolin E, et al. Phase 3 trial of ${ }^{177}$ Lu-Dotatate for midgut neuroendocrine tumors. N Engl J Med. 2017;376:125-135.

7. Brabander T, van der Zwan WA, Teunissen JJM, et al. Long-term efficacy, survival, and safety of $\left[{ }^{177} \mathrm{Lu}-\mathrm{DOTA}^{0}, \mathrm{Tyr}^{3}\right]$ octreotate in patients with gastroenteropancreatic and bronchial neuroendocrine tumors. Clin Cancer Res. 2017;23:4617-4624.

8. Gill MR, Falzone N, Du Y, Vallis KA. Targeted radionuclide therapy in combined-modality regimens. Lancet Oncol. 2017;18:e414-e423.

9. Lee ST, Kulkarni HR, Singh A, Baum RP. Theranostics of neuroendocrine tumors. Visc Med. 2017;33:358-366.

10. Zhang J, Wang H, Jacobson O, et al. Safety, pharmacokinetics, and dosimetry of a long-acting radiolabeled somatostatin analog ${ }^{177} \mathrm{Lu}$-DOTA-EB-TATE in patients with advanced metastatic neuroendocrine tumors. J Nucl Med. 2018;59:1699-1705.

11. Schultz MK, Mueller D, Baum RP, Leonard Watkins G, Breeman WA. A new automated $\mathrm{NaCl}$ based robust method for routine production of gallium-68 labeled peptides. Appl Radiat Isot. 2013;76:46-54.

12. Deppen SA, Liu E, Blume JD, et al. Safety and efficacy of ${ }^{68}$ Ga-DOTATATE PET/CT for diagnosis, staging, and treatment management of neuroendocrine tumors. J Nucl Med. 2016;57:708-714.

13. Wehrmann C, Senftleben S, Zachert C, Muller D, Baum RP. Results of individual patient dosimetry in peptide receptor radionuclide therapy with ${ }^{177} \mathrm{Lu}$ DOTATATE and ${ }^{177}$ Lu DOTA-NOC. Cancer Biother Radiopharm. 2007;22:406-416.

14. Bodei L, Mueller-Brand J, Baum RP, et al. The joint IAEA, EANM, and SNMMI practical guidance on peptide receptor radionuclide therapy (PRRNT) in neuroendocrine tumours. Eur J Nucl Med Mol Imaging. 2013;40:800-816.

15. Baum RP, Kulkarni HR. THERANOSTICS: from molecular imaging using Ga68 labeled tracers and PET/CT to personalized radionuclide therapy-the Bad Berka experience. Theranostics. 2012;2:437-447.

16. Schuchardt C, Kulkarni HR, Prasad V, Zachert C, Muller D, Baum RP. The Bad Berka dose protocol: comparative results of dosimetry in peptide receptor radionuclide therapy using ${ }^{177} \mathrm{Lu}$-DOTATATE, ${ }^{177} \mathrm{Lu}$-DOTANOC, and ${ }^{177} \mathrm{Lu}$-DOTATOC. Recent Results Cancer Res. 2013;194:519-536.

17. Kulkarni HR, Schuchardt C, Baum RP. Peptide receptor radionuclide therapy with ${ }^{177} \mathrm{Lu}$ labeled somatostatin analogs DOTATATE and DOTATOC: contrasting renal dosimetry in the same patient. Recent Results Cancer Res. 2013;194:551-559.

18. Bodei L, Kidd M, Paganelli G, et al. Long-term tolerability of PRRT in 807 patients with neuroendocrine tumours: the value and limitations of clinical factors. Eur J Nucl Med Mol Imaging. 2015;42:5-19.
19. Rufini V, Baum RP, Castaldi $P$, et al. Role of PET/CT in the functional imaging of endocrine pancreatic tumors. Abdom Imaging. 2012;37:1004-1020.

20. Baum RP, Kulkarni HR, Carreras C. Peptides and receptors in image-guided therapy: theranostics for neuroendocrine neoplasms. Semin Nucl Med. 2012; 42:190-207.

21. Pavel M, Baudin E, Couvelard A, et al. ENETS consensus guidelines for the management of patients with liver and other distant metastases from neuroendocrine neoplasms of foregut, midgut, hindgut, and unknown primary. Neuroendocrinology. 2012;95:157-176.

22. Eisenhauer EA, Therasse P, Bogaerts J, et al. New response evaluation criteria in solid tumours: revised RECIST guideline (version 1.1). Eur J Cancer. 2009; 45:228-247.

23. Khan S, Krenning EP, van Essen M, Kam BL, Teunissen JJ, Kwekkeboom DJ. Quality of life in 265 patients with gastroenteropancreatic or bronchial neuroendocrine tumors treated with $\left[{ }^{177} \mathrm{Lu}-\mathrm{DOTA}^{0}, \mathrm{Tyr}^{3}\right]$ octreotate. J Nucl Med. 2011;52:1361-1368.

24. Kwekkeboom DJ, Teunissen JJ, Bakker WH, et al. Radiolabeled somatostatin analog $\left[{ }^{177} \mathrm{Lu}-\mathrm{DOTA}{ }^{0}, \mathrm{Tyr}^{3}\right]$ octreotate in patients with endocrine gastroenteropancreatic tumors. J Clin Oncol. 2005;23:2754-2762.

25. Smit Duijzentkunst DA, Kwekkeboom DJ, Bodei L. Somatostatin receptor 2targeting compounds. J Nucl Med. 2017;58(suppl 2):54S-60S.

26. Ehlerding EB, Lan X, Cai W. "Albumin hitchhiking" with an Evans blue analog for cancer theranostics. Theranostics. 2018;8:812-814.

27. Cremonesi M, Ferrari ME, Bodei L, et al. Correlation of dose with toxicity and tumour response to ${ }^{90} \mathrm{Y}$ - and ${ }^{177} \mathrm{Lu}$-PRRT provides the basis for optimization through individualized treatment planning. Eur J Nucl Med Mol Imaging. 2018; 45:2426-2441.

28. Armaghany T, Vahdati G, Thamake S, Hamidi M, Amerinia R, Delpassand E. Treatment of high grade metastatic neuroendocrine tumor (mNET) with peptide receptor radionuclide therapy (PRRT): retrospective analysis in a single referral center. J Clin Oncol. 2017;33(15 suppl):e15175.

29. Nicolini S, Severi S, Ianniello A, et al. Investigation of receptor radionuclide therapy with ${ }^{177} \mathrm{Lu}$-DOTATATE in patients with GEP-NEN and a high Ki-67 proliferation index. Eur J Nucl Med Mol Imaging. 2018;45:923-930.

30. Hicks RJ, Kwekkeboom DJ, Krenning E, et al. ENETS consensus guidelines for the standards of care in neuroendocrine neoplasia: peptide receptor radionuclide therapy with radiolabeled somatostatin analogues. Neuroendocrinology. 2017;105: 295-309.

31. Baum RP, Kluge AW, Kulkarni HR, et al. $\left[{ }^{177} \text { Lu-DOTA }\right]^{0}$-D-Phe ${ }^{1}-$ Tyr $^{3}$-octreotide $\left({ }^{177} \mathrm{Lu}\right.$-DOTATOC) for peptide receptor radiotherapy in patients with advanced neuroendocrine tumours: a phase-II study. Theranostics. 2016;6:501-510.

32. Sorbye H, Welin S, Langer SW, et al. Predictive and prognostic factors for treatment and survival in 305 patients with advanced gastrointestinal neuroendocrine carcinoma (WHO G3): the NORDIC NEC study. Ann Oncol. 2013;24:152-160.

33. Binderup T, Knigge U, Loft A, Federspiel B, Kjaer A. ${ }^{18} \mathrm{~F}$-fluorodeoxyglucose positron emission tomography predicts survival of patients with neuroendocrine tumors. Clin Cancer Res. 2010;16:978-985.

34. Severi S, Nanni O, Bodei L, et al. Role of ${ }^{18}$ FDG PET/CT in patients treated with ${ }^{177}$ Lu-DOTATATE for advanced differentiated neuroendocrine tumours. Eur J Nucl Med Mol Imaging. 2013;40:881-888.

35. Kratochwil C, Stefanova M, Mavriopoulou E, et al. SUV of $\left[{ }^{68} \mathrm{Ga}\right]$ DOTATOCPET/CT predicts response probability of PRRT in neuroendocrine tumors. Mol Imaging Biol. 2015;17:313-318. 